\title{
Investigación, base de la innovación y eje de la competitividad empresarial
}

\author{
Lic. MBA. Gloria LOPEZ RIVAS
}

\section{INTRODUCCIÓN}

En este artículo trataré sobre la investigación como pivote alrededor del cual gira la innovación. Llamamos innovación a: "nuevos productos, procesos o servicios, e incluso a formas organizacionales y de comercialización que el mercado valora y que, por tanto, permiten incrementar la competitividad de las empresas y la calidad de vida de los ciudadanos" en una realidad económica y geográfica específica. La innovación constituye un eje que debe sustentar la competitividad de las empresas peruanas, siendo una tendencia que emerge con gran fuerza del entorno complejo e incierto que hoy predomina.

\section{SITUACIÓN DE LA RELACIÓN ENTRE INNOVACIÓN E INVESTI- GACIÓN}

La serie de cambios que condicionan el entorno actual ocasionan gran incertidumbre debido a las graves crisis del modelo Neo liberal y de la globalización de la actividad económica. Por ello, las empresas se ven forzadas a competir teniendo como pivote a la "innovación," la cual es fruto del avance del conocimiento. Ella se vincula así mismo con el acelerado cambio en el ciclo de los negocios. Esto ocasiona rupturas en la empresa. De acuerdo con Piore Sabelle (1990) en su obra" La Segunda Ruptura Industrial" (1) se postula que hay cambios en las relaciones entre empresas, en la propia organización interna de las mismas, donde las estructuras organizacionales tienden a ser más planas, con una continua redefinición e integración de sus áreas funcionales. La tecnología fuerza la reconversión de la producción. Se busca reducir cuellos de botella, tiempos muertos, mermas, errores, stock de inventarios, tiempos de producción y de entrega, acrecentando al mismo tiempo la pertinencia y la calidad. De otro lado, como resultado de la explosión tecnológica, se esta cambiando todo el sentido de la organización del trabajo: la misma noción de tarea y de puesto ha variado; hoy se busca actuar no solo sobre el contenido sino sobre las desviaciones imprevistas e incidentes. Como resultado, hay cambios en la comunicación, aparece el trabajador versátil y la exigencia de que los 
trabajadores sean multicualificados. De acuerdo a autores recientes, la empresa combina tecnología, conocimiento y capacidades que se generan y amplifican a través de aprendizajes colectivos y están sometidas a exigencias de adaptación, innovación y "uniqueness"o sea de diferenciación continua, que constituyen un desafío para todas las empresas, y en particular para las Pymes.

En el meollo de estos cambios esta la necesidad de innovar a través de la investigación que crea "nuevo conocimiento" no solo para crear nuevos productos o mejorar procesos sino también para adelantarse a los problemas y buscarles solución. En esta función las grandes empresas cumplen un rol vital por ser los agentes que realizan la investigación y el desarrollo (I \& D) mediante inversiones que hacen sea en adquisición de nuevas tecnologías o en la adaptación de estas a nuestro particular contexto social, económico y ambiental.

De acuerdo a un estudio del BID (2), el Perú exhibe un gran rezago en cuanto al desarrollo de Ciencia y Tecnología. Si bien el Perú ocupa el puesto $78^{a}$ dentro del ranking de Competitividad Global 2009-2010, tiene su talón de Aquiles en Infraestructura, Instituciones y en Innovación, siendo esta ultima la más crítica. En este aspecto el Perú ocupa el lugar 109 entre 133 países reflejando su bajo nivel en este campo entre los países de Latinoamérica, lo que se evidencia en el hecho que apenas invertimos en investigación y desarrollo solo el 0.15 de nuestro PBI.

De acuerdo a los indicadores de Innovación reflejados en el Gasto en Ciencia y Tecnología con respecto del PBI y al Coeficiente de Invención, el cuadro $\mathrm{N}^{\mathrm{o}} 1$, muestra que nuestro País tiene un desempeño muy pobre situándose muy por debajo del promedio de los países de América Latina.

\section{Cuadro $N^{0}$ 1: Gasto en ciencia y tecnología por sector de ejecución}

\begin{tabular}{|c|c|c|c|c|c|c|}
\hline & & 1993 & 2003 & 2004 & 2007 & 2008 \\
\hline \multirow{8}{*}{ Perú } & Gobierno & $25,6 \%$ & $26,0 \%$ & & & \\
\hline & Empresas & $6,5 \%$ & $4,3 \%$ & & & \\
\hline & Educación superior & $60,2 \%$ & $60,3 \%$ & & & \\
\hline & $\begin{array}{l}\text { Org. Priv. Sin fines de } \\
\text { lucro }\end{array}$ & $7,7 \%$ & $9,4 \%$ & & & \\
\hline & Gobierno & & $35,4 \%$ & $25,6 \%$ & & \\
\hline & Empresas & & $9,8 \%$ & $29,2 \%$ & & \\
\hline & Educación superior & & $44,7 \%$ & $38,1 \%$ & & \\
\hline & $\begin{array}{l}\text { Org. Priv. Sin fines de } \\
\text { lucro }\end{array}$ & & $10,1 \%$ & $7,1 \%$ & & \\
\hline
\end{tabular}




\begin{tabular}{|l|l|l|l|l|l|l|}
\hline & Gobierno & $28,5 \%$ & $30,9 \%$ & $29,7 \%$ & & \\
\cline { 2 - 7 } & Empresas & $21,1 \%$ & $25,5 \%$ & $26,2 \%$ & & \\
\hline & Educación superior & $49,3 \%$ & $41,3 \%$ & $42,3 \%$ & & \\
\hline $\begin{array}{l}\text { América } \\
\text { Latina y } \\
\text { el Caribe }\end{array}$ & $\begin{array}{l}\text { Org. Priv. Sin fines de } \\
\text { lucro }\end{array}$ & $1,0 \%$ & $2,3 \%$ & $1,9 \%$ & & \\
\cline { 2 - 7 } & Gobierno & $19,7 \%$ & $24,3 \%$ & $23,1 \%$ & & \\
\cline { 2 - 7 } & Empresas & $23,4 \%$ & $35,5 \%$ & $40,1 \%$ & & \\
\cline { 2 - 7 } & Educación superior & $54,6 \%$ & $37,9 \%$ & $34,8 \%$ & & \\
\hline & $\begin{array}{l}\text { Org. Priv. Sin fines de } \\
\text { lucro }\end{array}$ & $2,2 \%$ & $2,3 \%$ & $2,0 \%$ & & \\
\hline $\begin{array}{l}\text { Notas: } \\
\text { Los datos de América Latina y el Caribe son estimados } \\
\text { ACT: Actividades científicas y tecnológicas } \\
\text { I+D: Investigación y desarrollo }\end{array}$
\end{tabular}

Fuente: RICYT (2008) "El estado de la ciencia”.(3)

Respecto al déficit institucional es necesario que se cree un sistema público efectivo, que tome decisiones acertadas y reduzca significativamente las colas, retrasos y trámites burocráticos a fin de reducir los costos de atención. Debemos incorporar a las entidades privadas representativas y tanto a nivel de gremios como de las empresas, se debe fortalecer las capacidades y construir competencias. En cuanto a innovación tecnológica hay que incrementar y acelerar la aplicación del conocimiento con el fin de mejorar substancialmente la calidad e incrementar la productividad usando las herramientas que hoy provee la Ciencia, la Tecnología y la Innovación.

\section{ESTADO DE LA INNOVACIÓN EN EL PERÚ}

En un diagnóstico del estado de la Innovación en el Perú actual, se han detectado tendencias marcadas, destacando las siguientes:

1.-Son las grandes empresas las que muestran la mayor propensión "a innovar" y traer nueva tecnología. El cuadro $\mathrm{N}^{\circ} 2$, muestra que el $100 \%$ de las empresas dedicadas al sector Electricidad, Gas y agua; el 94\% de la Construcción, el 87\% de Inmobiliarias y el 79.5\% de las de Servicios prestados a empresas, fueron las responsables de haber introducido cambios tecnológicos. También nos muestra que hay una propensión a innovar, sin importar el tamaño de las empresas. Las empresas que tienen una poca o nula propensión a innovar son, las vinculadas a la pesca, comercio, restaurantes, Hoteles y transportes.

2.- Son múltiples las razones que inducen a las empresas a buscar e implantar nuevas tecnologías. El 57\% de empresas de Lima Metropolitana respondieron 
que a este respecto, la fuerza impulsora fue "mejorar la calidad de su producto o de su proceso productivo". El 41\% contestó que lo hizo por "reducir Costos", y los restantes para incrementar su producción. "Estas tres razones soportan la hipótesis que en nuestro País la innovación es reactiva, es decir se hace básicamente por la necesidad de competir o de enfrentar a la competencia global que va erosionando nuestro mercado. (Ver gráfico $\mathrm{N}^{\circ} 2$ )

Cuadro $N^{0}$ 2: Porcentaje de empresas que introdujeron cambios tecnológicos, según rama económica y tamaño de empresa en Lima metropolitana

\begin{tabular}{|c|c|c|c|c|}
\hline \multirow[b]{2}{*}{ Rama económica } & \multicolumn{4}{|c|}{ Tamaño de la empresa } \\
\hline & $\begin{array}{l}100 \text { a más } \\
\text { trabajadores }\end{array}$ & $\begin{array}{lr}50 & - \\
\text { trabajadores }\end{array}$ & 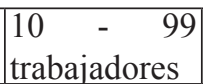 & total \\
\hline Pesca & & $33,3 \%$ & $71,4 \%$ & $63,9 \%$ \\
\hline Minería & $66,0 \%$ & $100,0 \%$ & $50,0 \%$ & $60,3 \%$ \\
\hline Industria & $66,8 \%$ & $60,0 \%$ & $50,1 \%$ & $53,8 \%$ \\
\hline Construcción & $94,9 \%$ & $75,0 \%$ & $41,2 \%$ & $50,1 \%$ \\
\hline Comercio & $66,6 \%$ & $40,8 \%$ & $44,7 \%$ & $45,8 \%$ \\
\hline Restaurantes y hoteles & $44,7 \%$ & $29,4 \%$ & $42,1 \%$ & $41,2 \%$ \\
\hline $\begin{array}{l}\text { Transporte, almacenamiento y } \\
\text { comunicaciones }\end{array}$ & $49,2 \%$ & $50,0 \%$ & $52,4 \%$ & $51,8 \%$ \\
\hline $\begin{array}{l}\begin{array}{l}\text { Establecimientos financieros y } \\
\text { seguros }\end{array} \\
\end{array}$ & $66,0 \%$ & $66,7 \%$ & $66,7 \%$ & $66,5 \%$ \\
\hline Servicios sociales y comunitarios & $38,5 \%$ & $85,7 \%$ & $60,0 \%$ & $60,9 \%$ \\
\hline Servicios prestados a empresas & $79,5 \%$ & $50,0 \%$ & $44,4 \%$ & $49,6 \%$ \\
\hline Actividades inmobiliarias & $58,7 \%$ & $0,0 \%$ & $66,7 \%$ & $59,8 \%$ \\
\hline Electricidad, gas y agua & $100,0 \%$ & & & $100,0 \%$ \\
\hline Total & $65,60 \%$ & $56,0 \%$ & $48,6 \%$ & $51,2 \%$ \\
\hline
\end{tabular}

Fuente: MTPE. Encuesta a empresas sobre el desarrollo de los recursos humanos, 2007

3.- El proceso de crecimiento del País exige una mejora importante en el esfuerzo competitivo empresarial, obligando a que una apreciable porción de empresas haya introducido cambios tecnológicos. Sin embargo hay problemas que se confrontan para implementar el proceso de innovación tecnológica. Entre estos, destacan: a) La falta de personal calificado b) la falta de acceso a Información así como de Asistencia Técnica. c) la falta de financiamiento. 
Cuadro $\mathrm{N}^{\circ}$ 3: Problemas de las empresas de Lima Metropolitana para implementar innovación tecnológica en el 2007.

\begin{tabular}{|l|l|l|l|l|}
\hline \multicolumn{5}{|l}{ Tamaño de la empresa } \\
\cline { 2 - 6 } & $\begin{array}{l}100 \text { a más } \\
\text { trabajadores }\end{array}$ & $\begin{array}{l}50-99 \\
\text { trabajadores }\end{array}$ & $\begin{array}{l}10-99 \\
\text { trabajadores }\end{array}$ & total \\
\hline Falta de personal calificado & $7,40 \%$ & $14,2 \%$ & $4,0 \%$ & $5,6 \%$ \\
\hline Falta de información & $9,9 \%$ & $1,9 \%$ & $4,6 \%$ & $5,0 \%$ \\
\hline Falta de asistencia técnica & $7,8 \%$ & $7,0 \%$ & $3,9 \%$ & $4,8 \%$ \\
\hline Tamaño reducido de la empresa & $2,3 \%$ & $2,0 \%$ & $2,8 \%$ & $2,6 \%$ \\
\hline $\begin{array}{l}\text { Situación económica desfavora- } \\
\text { ble de la empresa }\end{array}$ & $0,5 \%$ & $2,5 \%$ & $0,9 \%$ & $1,0 \%$ \\
\hline Dificultad de financiamiento & $3,9 \%$ & $7,9 \%$ & $4,6 \%$ & $4,8 \%$ \\
\hline $\begin{array}{l}\text { Problemas para la importación } \\
\text { de insumos o equipos }\end{array}$ & $1,2 \%$ & $0,0 \%$ & $0,0 \%$ & $0,2 \%$ \\
\hline
\end{tabular}

Otro gran problema es la necesidad de formación debido a la rapidez con que los conocimientos se obsolescen y la consiguiente necesidad de aprendizaje continuo. Así mismo, esta situación explica la fuga de talentos del Perú y la necesidad de entrenar equipos orientados a tareas desafiantes, ya que la naturaleza y la complejidad de las tareas se van acrecentando. Por consiguiente, resulta que más allá de la adaptación a los puestos de trabajo, se requiere "saber Hacer", es decir, desarrollar competencias para afrontar de manera original las nuevas oportunidades que se presentan en los lugares de trabajo.

En conclusión si bien el Perú ha ido mejorando en su posición competitiva, todavía está en una situación muy deficitaria en materia de innovación. Según los expertos está en emergencia, ya que ocupa la posición $\mathrm{N}^{\mathrm{o}} 110$ dentro del ranking de competitividad mundial en innovación, muy por debajo de Brasil, Chile y Colombia.

\section{QUÉ HACER PARA MEJORAR LA INNOVACIÓN EN EL PAÍS}

Para mejorar en innovación nuestro país deberá diseñar una nueva estrategia para generar y difundir nuevos conocimientos. En este tema el sector público exhibe una gran debilidad, el marco institucional es muy débil y hay una falta de protagonismo de la empresa privada, todos los indicadores así lo evidencian. Si bien hay indicios que las empresas, especialmente las grandes tienden a invertir en innovación por razones de reducción de costos, enfrentar a la competencia e incrementar su producción, la gran cantidad de empresas medianas y pequeñas (PYME) no lo hacen.

CÓmo iniciar la carrera de la innovación es un tema digno de investigación. Algunos expertos proponen que debiéramos empezar por el agro, la biotecno- 
logía aplicada a plantas, animales y minerales, y la innovación de los diversos procesos industriales.

Queda también pendiente el tema de qué incentivos debieran de dar tanto el Estado como las empresas para repatriar los talentos peruanos que existen en el extranjero.

Finalmente debo señalar que, para que el Perú, de el salto en la innovación, y potencie el valor económico de mediano y largo plazo convirtiéndose así en un país competitivo, debe asumir la serie de riesgos inherentes a la innovación. Esto implica estrategias para iniciar y mantener cambios sostenibles relativos a: a) los sistemas de gestión de la administración pública y privada b) la mejora permanente e instauración de nuevos métodos productivos en la agricultura, en los sectores industriales y de servicio, c) en el diseño y comercialización de productos que le permitan introducir productos con alto valor agregado en los mercados mundiales. Asimismo desde el punto de vista gerencial sería muy conveniente seguir la estrategia de los "océanos azules" que, como explicó Renèe Mauborgne, una de las expertas más influyentes sobre este tipo de estrategias, ésta demanda un enfoque novedoso basado en creatividad, donde la estrategia es la que moldea la estructura y donde la competencia se vuelve irrelevante. Lo más interesante de la propuesta mencionada es que lo mejor "es buscar un mercado que nadie haya tocado y que tenga un potencial de crecimiento a partir de una diferenciación y del bajo costo" (4).

Todos estos cambios requieren que los gerentes de empresas induzcan comportamientos que desafíen los paradigmas que dominan actualmente la industria, cambien sus modelos de negocios e internalicen la creatividad como manera de pensar cotidiana.

Estos cambios demandan políticas activas, instrumentos, incentivos públicos, y mecanismos financieros para el acceso al conocimiento, la innovación, el uso de la ciencia y la tecnología para la producción y el empleo. De otro lado, ya se han empezado a dar lineamientos de política (Ministerio de la Producción) que promueven la innovación tecnológica como factor de competitividad. A ello se ha añadido el diseño de los CITE, que son centros de Innovación tecnológica dentro de redes con un énfasis en las Pymes. Los CITE son instituciones de soporte tecnológico para las empresas y que facilitan en ellas las innovaciones y la trasferencia tecnológica que permitirán añadir valor y asegurar el cumplimiento de las normas técnicas, las buenas prácticas y otros estándares de calidad e higiene que exigen los mercados globales.

Se han constituido tres cites públicos con directorios privados los cuales representan alianzas entre la Empresa, el Estado y la Universidad. Estos cites 
son: el CITE cal, el CITE madera, Y el Cite vid. Además, el Ministerio de la Producción cuenta con la Oficina CITE-OTCIT encargada de fomentar la innovación, la transferencia de tecnología y alianzas estratégicas dentro y fuera del País. La relación de los CITES vigentes es la siguiente(5).

\begin{tabular}{|c|c|c|c|}
\hline CITE & $\begin{array}{l}\text { Fecha de } \\
\text { acreditación }\end{array}$ & $\begin{array}{l}\text { ¿Qué cadenas } \\
\text { productivas atiende? }\end{array}$ & $\begin{array}{l}\text { ¿Quiénes lo } \\
\text { conforman? }\end{array}$ \\
\hline CITE CAL-LIMA & Julio de 1998 & $\begin{array}{l}\text { Cuero, calzado e } \\
\text { industrias conexas }\end{array}$ & $\begin{array}{l}\text { CITE público, } \\
\text { PRODUCE. Su } \\
\text { consejo directivo } \\
\text { está integrado } \\
\text { por empresarios } \\
\text { y funcionarios de } \\
\text { PromPeru } \\
\end{array}$ \\
\hline CITE VID-ICA & Octubre del 2000 & $\begin{array}{l}\text { Vitinicola / } \\
\text { agroindustrial }\end{array}$ & $\begin{array}{l}\text { CITE público, } \\
\text { depende de } \\
\text { PRODUCE. Su } \\
\text { directiva está } \\
\text { conformada por } \\
\text { empresarios líderes, } \\
\text { PromPeru y MINAG }\end{array}$ \\
\hline $\begin{array}{l}\text { CITE MADERA- } \\
\text { SEDE CENTRAL } \\
\text { EN LIMA } \\
\text { Y UNIDAD } \\
\text { TÉCNICA EN } \\
\text { PUCALLPA }\end{array}$ & Octubre del 2000 & Madera y muebles & $\begin{array}{l}\text { CITE público, } \\
\text { depende de } \\
\text { PRODUCE. Su } \\
\text { directiva está } \\
\text { conformada por } \\
\text { empresarios líderes y } \\
\text { PromPeru y MINAG }\end{array}$ \\
\hline $\begin{array}{l}\text { CITE FRUTAS } \\
\text { TROPICALES } \\
\text { Y PLANTAS } \\
\text { MEDICINALES DE } \\
\text { LORETO }\end{array}$ & Mayo del 2002 & $\begin{array}{l}\text { Frutas tropicales( } \\
\text { Camú Camú, Sacha } \\
\text { Incha) y plantas } \\
\text { medicinales( uña } \\
\text { de gato sangre de } \\
\text { grado) }\end{array}$ & $\begin{array}{l}\text { CITE privado, } \\
\text { integrado por } \\
\text { la Universidad } \\
\text { Nacional de la } \\
\text { Amazonía Peruana, } \\
\text { IIEP, EsSalud, IMET, } \\
\text { Gobierno Regional } \\
\text { De Loreto, Cámara } \\
\text { de Comercio e IPPN }\end{array}$ \\
\hline $\begin{array}{l}\text { CITE } \\
\text { CONFECCIONES } \\
\text { EL TALLER - } \\
\text { AREQUIPA } \\
\end{array}$ & Julio del 2002 & $\begin{array}{l}\text { Confecciones de } \\
\text { tejido y plano y de } \\
\text { punto }\end{array}$ & $\begin{array}{l}\text { CITE privado, } \\
\text { conformado por la } \\
\text { ONG y El taller de } \\
\text { Arequipa }\end{array}$ \\
\hline $\begin{array}{l}\text { CITE AGROIN- } \\
\text { DUSTRIAL MST- } \\
\text { TACNA }\end{array}$ & Mayo del 2003 & $\begin{array}{l}\text { Agroindustrial: } \\
\text { olivícola, ají, } \\
\text { hierbas aromáticas, } \\
\text { hortofrutícola y } \\
\text { vitivinícola }\end{array}$ & $\begin{array}{l}\text { CITE privado, } \\
\text { conformado por } \\
\text { PRODUCE, } \\
\text { ZOFRATACNA, } \\
\text { Gobierno regional de } \\
\text { Tacna y empresarios } \\
\text { de olivo vitinícola ají } \\
\text { y orégano. }\end{array}$ \\
\hline
\end{tabular}




\begin{tabular}{|c|c|c|c|}
\hline $\begin{array}{l}\text { CITE AGROIN- } \\
\text { DUSTRIAL } \\
\text { CEPRORUI - } \\
\text { AREQUIPA }\end{array}$ & Junio del 2003 & $\begin{array}{l}\text { Hierbas aromáticas } \\
\text { orgánicas y } \\
\text { otros productos } \\
\text { hortofrutícolas. }\end{array}$ & $\begin{array}{l}\text { CITE privado, } \\
\text { conformado por } \\
\text { la ONG, El taller } \\
\text { de Arequipa en } \\
\text { convenio con } \\
\text { AUTODEMA } \\
\text { (GRA) }\end{array}$ \\
\hline $\begin{array}{l}\text { CITE } \\
\text { INDUSTRIAL } \\
\text { TEXTIL } \\
\text { CAMÉLIDOS } \\
\text { DEL PERÚ IPAC - } \\
\text { AREQUIPA }\end{array}$ & Noviembre del 2003 & $\begin{array}{l}\text { Confecciones textil } \\
\text { camélidos }\end{array}$ & $\begin{array}{l}\text { CITE privado, } \\
\text { conformado por el } \\
\text { Instituto Peruano } \\
\text { de Alpaca (IPAC), } \\
\text { que integra a } \\
\text { productores } \\
\text { alpaqueros, } \\
\text { industriales } \\
\text { textiles y pequeños } \\
\text { empresarios } \\
\text { confeccionistas. }\end{array}$ \\
\hline $\begin{array}{l}\text { CITE AGROIN- } \\
\text { DUSTRIAL PIURA }\end{array}$ & Agosto del 2004 & $\begin{array}{l}\text { Agroindustrial: } \\
\text { mango, banano, } \\
\text { café, panela y } \\
\text { algarrobina }\end{array}$ & $\begin{array}{l}\text { CITE privado, } \\
\text { integrado por } \\
\text { la Universidad } \\
\text { de Piura, la } \\
\text { asociación regional } \\
\text { de Productores } \\
\text { de Algarrobina- } \\
\text { PIUERA (ARPAL), } \\
\text { La Cámara } \\
\text { de Comercio, } \\
\text { PROMANGO, } \\
\text { Centro Ecuménico } \\
\text { de Promoción y } \\
\text { Acción Social del } \\
\text { Norte (CEDEPAS } \\
\text { NORTE), } \\
\text { PRODUCE y } \\
\text { CEPICAFE. }\end{array}$ \\
\hline $\begin{array}{l}\text { CITE LOGÍSTICA } \\
\text { GS1 }\end{array}$ & Setiembre del 2006 & $\begin{array}{l}\text { Transversal a } \\
\text { todas las cadenas } \\
\text { productivas en el } \\
\text { tema logístico }\end{array}$ & $\begin{array}{l}\text { CITE privado, } \\
\text { conformado por } \\
\text { GS1 Perú }\end{array}$ \\
\hline CITE SOFTWARE & Marzo del 2007 & $\begin{array}{l}\text { Transversal a todas } \\
\text { las de cadenas } \\
\text { productivas en el } \\
\text { tema software }\end{array}$ & $\begin{array}{l}\text { CITE privado, } \\
\text { conformado } \\
\text { por APESOFT } \\
\text { (Asociación Peruana } \\
\text { de Empresas de } \\
\text { Software) }\end{array}$ \\
\hline
\end{tabular}




\begin{tabular}{|l|l|l|l|}
\hline $\begin{array}{l}\text { CENTRO DE } \\
\text { INNOVACIÓN } \\
\text { TECNOLÓGICA } \\
\text { AGROINDUS- } \\
\text { TRIAL DE MAJES } \\
\text { Y EL SUR DEL } \\
\text { PERÚ- CITE LÁC- } \\
\text { TEOS }\end{array}$ & Febrero del 2008 & $\begin{array}{l}\text { Agroalimentario, } \\
\text { específicamente en } \\
\text { lácteos }\end{array}$ & $\begin{array}{l}\text { CITE privado, } \\
\text { conformado por } \\
\text { IDESI-Arequipa } \\
\text { en convenio con } \\
\text { universidades del } \\
\text { Sur. }\end{array}$ \\
\hline $\begin{array}{l}\text { CITE } \\
\text { CONFECCIONES } \\
\text { Y DISEÑO DE } \\
\text { MODA }\end{array}$ & Julio del 2009 & Textil confecciones & $\begin{array}{l}\text { CITE privado, } \\
\text { conformado por } \\
\text { la Corporación } \\
\text { CHIO LECCA } \\
\text { Capacitación y } \\
\text { Consultoría SAC }\end{array}$ \\
\hline
\end{tabular}

\section{REFERENCIAS BIBLIOGRÁFICAS}

1. Piore Sabelle (1990) en su obra" La Segunda Ruptura Industrial".

2. Banco Interamericano de Desarrollo: "Temas en Competitividad, Tecnologia e Innovacion": BID 2012. http://www.iadb.org/es/temas/competitividadtecnologia-e-innovacion/mapa-de-proyectos,2844.html.

3. RICYT (2008) “El estado de la ciencia”: www.vinv.ucr.ac.cr/docs/ divulgacion-ciencia/.../el-estado-ciencia-2009.pdf

4. EKOS Hablemos de Innovación. N $N^{\circ}$ 7. Julio-Agosto 2013. Una Estrategia Innovadora por Renèe Mauborgne.

5. Red de CITES promovidos por PRODUCE: http://ftp.produce.gob.pe/ RepositorioAPS/2/jer/SERVCITE/cites/directorio_cites.pdf 
